\title{
Parity Violation in Neutrino Transport and the Origin of Pulsar Kicks
}

\author{
Dong Lai \\ Theoretical Astrophysics, California Institute of Technology, Pasadena, CA 91125; \\ Department of Astronomy, Cornell University, Ithaca, NY 14853 \\ E-mail: dong@spacenet.tn.cornell.edu \\ and \\ Yong-Zhong Qian \\ Physics Department, 161-33, California Institute of Technology, Pasadena, CA 91125
}

\begin{abstract}
In proto-neutron stars with strong magnetic fields, the neutrino-nucleon scattering/absorption cross sections depend on the direction of neutrino momentum with respect to the magnetic field axis, a manifestation of parity violation in weak interactions. We study the deleptonization and thermal cooling (via neutrino emission) of proto-neutron stars in the presence of such asymmetric neutrino opacities. Significant asymmetry in neutrino emission is obtained due to multiple neutrino-nucleon scatterings. For an ordered magnetic field threading the neutron star interior, the fractional asymmetry in neutrino emission is about $0.006\left(B / 10^{14} \mathrm{G}\right)$, corresponding to a pulsar kick velocity of $214\left(B / 10^{14} \mathrm{G}\right) \mathrm{km} \mathrm{s}^{-1}$ for a total radiated neutrino energy of $3 \times 10^{53} \mathrm{erg}$. This represents the most efficient way to generate pulsar kicks using asymmetric neutrino emission induced by magnetic fields.
\end{abstract}

Subject headings: stars: neutron - pulsars: general - stars: supernova - neutrinos dense matter

\section{Introduction}

Recent analyses of pulsar proper motion (Lyne \& Lorimer 1994; Lorimer et al. 1997; Hansen \& Phinney 1997; Cordes \& Chernoff 1997), measurements of interstellar scintillation (Cordes 1986; Harrison \& Lyne 1993), and studies of pulsar-supernova remnant associations (Frail et al. 1994) indicate that supernova explosions are asymmetric and the neutron stars receive large kick velocities at birth $\left(250-500 \mathrm{~km} \mathrm{~s}^{-1}\right.$ on average, but possibly with a significant population having velocities greater than $1000 \mathrm{~km} \mathrm{~s}^{-1}$ ). Compelling evidence for supernova asymmetry also comes from the detection of geodetic precession in PSR 1913+16 (Cordes et al. 1990) and orbital plane precession in the PSR J0045-7319/B star binary (Lai et al. 1995; Kaspi et al. 1996), as well 
as numerous direct observations of nearby supernovae in radio, optical, and X-ray bands (e.g., McCray 1993; Panagia et al. 1996). In addition, evolutionary considerations of double neutron star binaries also imply the existence of pulsar kicks (e.g., Fryer \& Kalogera 1997).

Two classes of mechanisms for the natal kicks of pulsars have been suggested. The first class relies on local hydrodynamic instabilities in the proto-neutron stars (Burrows et al. 1995; Burrows \& Hayes 1996; Janka \& Müller 1996; Herant et al. 1994) as well as global asymmetric perturbations seeded in the presupernova cores (Goldreich et al. 1996). The asymmetries in matter and temperature distributions naturally lead to asymmetric explosion and/or asymmetric neutrino emission, although the magnitude of the resulting kick velocity is unclear at this point.

In this letter, we focus on the second class of models in which the large pulsar velocities arise from asymmetric neutrino emission induced by strong magnetic fields. Although a number of papers have been written on this subject (Chugai 1984; Dorofeev et al. 1985; Binovatyi-Kogan 1993; Vilenkin 1995; Horowitz \& Piekarewicz 1997), they are unsatisfactory for a number of reasons: they either failed to identify the most relevant neutrino emission/interaction processes or the relevant physical conditions in proto-neutron stars, or stopped at estimating the magnetic field effects on neutrino opacities (some of these papers contain errors), and none of them treated neutrino transport in any systematic manner. As a result, their estimates of the kick velocity that may be produced are unreliable. We have carried out systematic studies of neutrino transport in strong magnetic fields (Lai \& Qian 1997a,b) in order to pin down the magnitude of the magnetic field effects on neutrino transport and pulsar kicks. Here we report our most important finding on the macroscopic consequence of asymmetric neutrino opacities due to parity violation in weak interactions.

The existence of parity violation effects in neutrino opacities relevant for proto-neutron stars has been noted by Chugai (1984) and Vilenkin (1995), who concluded that these effects are extremely small']. An important point was made by Horowitz \& Li (1997), who suggested that the neutrino asymmetry may be enhanced due to multiple scatterings of neutrinos by nucleons slightly polarized by the magnetic field. However, the paper by Horowitz \& Li (1997) does not contain a proper treatment of neutrino transport, and does not consider the effect of neutrino absorption which one might suspect to smear out the cumulative effect from multiple scatterings. In this letter, we present the first calculation of the thermal evolution of proto-neutron stars in the presence of asymmetric neutrino opacities. We demonstrate the existence of the cumulative effect from multiple scatterings even in the presence of neutrino absorption. We find that while the idea of Horowitz \& Li (1997) is basically correct, they greatly overestimated the magnitude of the asymmetric neutrino flux and the resulting kick velocity.

Our paper is organized as follows. In $\S 1$ we summarize the main asymmetric features in

\footnotetext{
${ }^{1}$ Both papers considered neutrino-electron scattering, which is less important than neutrino-nucleon scattering (see $\S 2$ and LQ). Note that Chugai's expression for the electron polarization in the relativistic degenerate regime (the relevant regime) is incorrect.
} 
neutrino opacities relevant for proto-neutron stars. Section 3 includes a brief discussion of the toy problem which we have studied to demonstrate the cumulative effect from multiple neutrino scatterings. In $\S 4$ we outline the derivation of neutrino transport equations with asymmetric opacities, and present our numerical results. A brief summary is given in $\S 5$. More details can be found in our forthcoming publication (Lai \& Qian 1997a, hereafter LQ).

\section{Parity Violation and Asymmetric Neutrino Opacities}

In the presence of an external magnetic field, the differential opacity (in $\mathrm{cm}^{2} \mathrm{~g}^{-1}$ ) for neutrino-nucleon scattering, $\nu_{(e, \mu, \tau)}+N \rightarrow \nu_{(e, \mu, \tau)}+N$, is asymmetric, and can be written in the form:

$$
\left(\frac{d \tilde{\kappa}_{E}^{(\mathrm{sc})}}{d \Omega^{\prime}}\right)_{\hat{\mathbf{\Omega}} \rightarrow \hat{\boldsymbol{\Omega}}^{\prime}}=\frac{1}{4 \pi} \kappa_{E}^{(\mathrm{sc})}\left[1+\epsilon_{\mathrm{in}} \hat{\boldsymbol{\Omega}} \cdot \hat{\mathbf{B}}+\epsilon_{\mathrm{out}} \hat{\mathbf{\Omega}}^{\prime} \cdot \hat{\mathbf{B}}+\mathrm{const} \times\left(\hat{\boldsymbol{\Omega}} \cdot \hat{\mathbf{\Omega}}^{\prime}\right)\right],
$$

where $\hat{\boldsymbol{\Omega}}$ and $\hat{\boldsymbol{\Omega}}^{\prime}$ are the unit momentum vectors for the incoming and outgoing neutrinos, respectively, $\hat{\mathbf{B}}$ is the unit vector along the magnetic field direction, and $\kappa_{E}^{(\mathrm{sc})}$ is the total scattering opacity without magnetic field (the subscript "E" implies that the opacity depends on the neutrino energy $E$ ). The last term (proportional to $\hat{\boldsymbol{\Omega}} \cdot \hat{\mathbf{\Omega}}^{\prime}$ ) inside the square bracket in Eq. (1) corresponds to the usual angular dependence for scattering. The presence of the $\hat{\mathbf{B}}$-depedent terms in Eq. (1) implies that there is a preferred direction in the opacity. This is a manifestation of parity violation in weak interactions. The coefficients $\epsilon_{\text {in }}$ and $\epsilon_{\text {out }}$ are related to the nucleon polarization $P$ by (LQ)

$$
\epsilon_{\mathrm{in}}=2 P \frac{c_{A}\left(c_{A}-c_{V}\right)}{c_{V}^{2}+3 c_{A}^{2}}, \quad \epsilon_{\text {out }}=-2 P \frac{c_{A}\left(c_{A}+c_{V}\right)}{c_{V}^{2}+3 c_{A}^{2}},
$$

where $c_{V}=-1 / 2, c_{A}=-1.15 / 2$ for neutron and $c_{V}=1 / 2-2 \sin ^{2} \theta_{W}=0.035, c_{A}=1.37 / 2$ for proton (Raffelt \& Seckel 1995). For nondegenerate nucleons, the spin polarization $P$ is

$$
P=\frac{\mu_{m} B}{k T}=3.15 \times 10^{-5} g_{m} B_{14}\left(\frac{10 \mathrm{MeV}}{T}\right),
$$

where $\mu_{m}$ is the nucleon magnetic moment $\left(g_{m}=-1.913\right.$ for neutron and 2.793 for proton), and $B_{14}$ is the field strength in units of $10^{14} \mathrm{G}$. In the degenerate regime, we have

$$
P=\frac{3 \mu_{m} B}{2 \mu_{N}}=4.73 \times 10^{-5} g_{m} B_{14}\left(\frac{10 \mathrm{MeV}}{\mu_{N}}\right),
$$

where $\mu_{N}$ is the nucleon chemical potential (or Fermi energy). A general expression for $P$ can also be derived (LQ). In proto-neutron stars, one should average Eq. (1) over composition (neutrons and protons). It is also important to note that the differential opacity for $\bar{\nu}_{e, \mu, \tau}$ scattering on nucleons is obtained by interchanging $\epsilon_{\mathrm{in}}$ and $\epsilon_{\text {out }}$ in Eq. (1) as a result of the crossing symmetry of the leading order matrix elements.

The neutrino absorption opacity is also asymmetric, i.e.,

$$
\tilde{\kappa}_{E}^{(\mathrm{abs})}=\kappa_{E}^{(\mathrm{abs})}\left(1+\epsilon_{a} \hat{\mathbf{\Omega}} \cdot \hat{\mathbf{B}}\right),
$$


with $\epsilon_{a} \sim P$. However, in the bulk interior of the neutron star, this asymmetry is exactly canceled by the asymmetry in emission (see $\S 4$ ). One also expects asymmetry in the inelastic scattering $\nu+e \rightarrow \nu+e$. However, for relativistic degenerate electrons, the $\nu$ - $e$ opacity is much smaller than the $\nu$-N opacity, while the electron polarization $P_{e} \simeq 2 \times 10^{-5} B_{14}\left(200 \mathrm{MeV} / \mu_{e}\right)^{2}$ is of the same order as $P$ for nucleons. Therefore the contribution from $\nu$-e scattering to asymmetric neutrino transport is small compared with that from $\nu-N$ scattering.

\section{Toy Problem: Effect of Multiple Scatterings}

The asymmetry coefficients $\left(\epsilon_{\text {in }}\right.$ and $\left.\epsilon_{\text {out }}\right)$ in the opacity are extremely small even at $B_{14}=10$. However, cumulative effect due to multiple scatterings can enhance the asymmetry in neutrino emission. To demonstrate this effect, we consider a simple toy problem in which neutrinos leak out of a slab (with infinite extent perpendicular to the $z$-axis) via biased diffusion, i.e., the neutrino scattering probability has the form $\propto(1+\epsilon \cos \theta)$, with $\epsilon \ll 1$ and $\theta$ being the angle between the $z$-axis (the magnetic field direction which is perpendicular to the slab) and the direction of neutrino propagation after each scattering. The evolution of the neutrino number density $n(z, t)$ is governed by the Fokker-Planck equation:

$$
\frac{\partial n}{\partial t}=\frac{1}{3} c \lambda \nabla^{2} n-\frac{1}{3} c \nabla \cdot(\epsilon n \hat{\mathbf{B}}),
$$

where $\lambda$ is the (constant) mean free path of the neutrino. The surfaces of the slab are located at $z= \pm \lambda \tau_{s}$ (so $2 \tau_{s}$ is the total optical depth of the slab). Suppose we start with a uniform distribution $n=n_{0}$ at $t=0$ (so that there is no initial asymmetry). As neutrinos gradually diffuse out, the profile $n(z, t)$ becomes increasingly asymmetric, and the flux asymmetry $\Delta F / F$ grows (where $F$ is the sum of the fluxes from both surfaces and $\Delta F$ is the difference). For $t \gtrsim \tau_{s}^{2}(\lambda / c)$, the "cooling" front reaches the center of the slab, and $\Delta F / F$ saturates to constant value, $\Delta F / F \simeq \epsilon \tau_{s} / 2$ (valid for $\epsilon \tau_{s} \ll 1$ ). This constant value can be derived analytically from a quasi-steady-state analysis (A general expression for arbitrary $\epsilon \tau_{s}$ can also be derived). The fractional asymmetry in the total neutrino radiation energy is $0.34 \epsilon \tau_{s}$. A similar toy problem for a cooling sphere has also been studied (LQ), showing the same physical effect (with slightly different numerical results).

In proto-neutron stars, the neutrino optical depth $\tau$ is large. A rough estimate can be made by setting the diffusion time $\sim 3 R^{2} /(\lambda c)$ ( $R$ is the neutron star radius) to $1 \mathrm{~s}$ (the spread in the arrival time of SN 1987A neutrinos was $10 \mathrm{~s}$ ), which gives $\tau \sim 10^{4}$. This would imply a large enhancement in neutrino emission asymmetry. Several complications arise when one applies the result of the toy problem to real neutron stars: As shown in $§ 4$, the asymmetry in neutrino flux is determined by the coefficient $\epsilon_{\text {out }}-\epsilon_{\text {in }}$ and the local neutrino energy distribution function. Due to the crossing symmetry, we have $\left(\epsilon_{\text {out }}-\epsilon_{\text {in }}\right)_{\nu}=-\left(\epsilon_{\text {out }}-\epsilon_{\text {in }}\right)_{\bar{\nu}}$. Because $\nu_{\mu(\tau)}$ and $\bar{\nu}_{\mu(\tau)}$ have the same local energy distribution function, there is no net asymmetry associated with $\nu_{\mu(\tau)}$ and

$\bar{\nu}_{\mu(\tau)}$ emission. The asymmetries in $\nu_{e}$ and $\bar{\nu}_{e}$ emission do not cancel because a newly-formed 
neutron star is lepton-rich, i.e., it contains more $\nu_{e}$ than $\bar{\nu}_{e}$, and the typical energy of $\nu_{e}$ is also different from $\bar{\nu}_{e}$. As the neutron star is deleptonized, the difference between $\nu_{e}$ and $\bar{\nu}_{e}$ becomes smaller, and we expect the asymmetry in the total neutrino flux to gradually diminish. Another issue which is not clear from the toy problem concerns the role of neutrino absorption: for electron-type neutrinos, absorption is more important than scattering (by a factor of a few) in the deleptonization phase. Does the neutrino absorption completely wipe out the asymmetry associated with multiple scatterings?

\section{Thermal Evolution of Proto-Neutron Stars with Asymmetric Neutrino Opacities}

We now describe our calculation of the thermal evolution of proto-neutron stars in the presence of the asymmetric neutrino opacities discussed in $\S 2$. We start from the general transport equation for the spectral intensity $I_{E}=I_{E}(\mathbf{r}, \hat{\boldsymbol{\Omega}}, t)$ of a given neutrino species:

$$
\begin{aligned}
& \frac{\partial I_{E}}{\partial t}+\hat{\mathbf{\Omega}} \cdot \nabla I_{E}=\rho \tilde{\kappa}_{E}^{(\mathrm{abs})}\left(I_{E}^{(F D)}-I_{E}\right)+\rho\left(1-f_{E}\right) \int\left(\frac{d \tilde{\kappa}_{E}^{(\mathrm{sc})}}{d \Omega}\right)_{\hat{\mathbf{\Omega}}^{\prime} \rightarrow \hat{\mathbf{\Omega}}} I_{E}^{\prime} d \Omega^{\prime} \\
& -\rho I_{E} \int\left(\frac{d \tilde{\kappa}_{E}^{(\mathrm{sc})}}{d \Omega^{\prime}}\right)_{\hat{\mathbf{\Omega}} \rightarrow \hat{\boldsymbol{\Omega}}^{\prime}}\left(1-f_{E}^{\prime}\right) d \Omega^{\prime},
\end{aligned}
$$

where $I_{E}^{\prime}=I_{E}\left(\mathbf{r}, \hat{\mathbf{\Omega}}^{\prime}, t\right), f_{E}=(2 \pi)^{3} I_{E} / E^{3}$ is the neutrino occupation number, and $I_{E}^{(F D)}$ is the Fermi-Dirac distribution function for neutrinos:

$$
I_{E}^{(F D)}(T)=\frac{c}{4 \pi} U_{E}^{(F D)}=\frac{E^{3}}{(2 \pi)^{3}} \frac{1}{e^{\left(E-\mu_{\nu}\right) / T}+1}
$$

$\left(U_{E}^{(F D)}\right.$ is the corresponding energy density), with matter temperature $T$ and neutrino chemical potential $\mu_{\nu}$. In Eq. (7) the absorption opacity $\tilde{\kappa}_{E}^{(\text {abs })}$ already includes the effect of stimulated absorption of neutrinos. Multiplying Eq. (7) by $\hat{\boldsymbol{\Omega}}$ and then integrating over $\hat{\boldsymbol{\Omega}}$, we obtain the first order moment equation:

$$
\mathbf{F}_{E}=-\frac{c}{3 \rho \kappa_{E}^{(t)}} \nabla U_{E}+\epsilon_{a} \frac{\kappa_{E}^{(\mathrm{abs})}}{3 \kappa_{E}^{(t)}} c\left(U_{E}^{(F D)}-U_{E}\right) \hat{\mathbf{B}}+\left(\epsilon_{\mathrm{out}}-\epsilon_{\mathrm{in}}\right) \frac{\kappa_{E}^{(\mathrm{sc})}}{3 \kappa_{E}^{(t)}} c U_{E}\left(1-f_{E}\right) \hat{\mathbf{B}},
$$

where $U_{E}, \mathbf{F}_{E}$ is the spectral energy density and flux, and $\kappa_{E}^{(t)}=\kappa_{E}^{(\mathrm{sc})}+\kappa_{E}^{(\mathrm{abs})}$ is the total opacity. The neutrino number flux is simply given by $\mathbf{S}_{E}=\mathbf{F}_{E} / E$. Equation (9) reveals that in addition to the usual "diffusive" flux (the first term), there is a "drift" flux (the $\hat{\mathbf{B}}$-dependent terms) along the magnetic field direction due to the asymmetric neutrino opacities. Note that when $U_{E}=U_{E}^{(F D)}$ (i.e., when neutrinos are in thermal equilibrium with matter), the asymmetric absorption opacity does not contribute to the drift flux, since in this case asymmetric emission exactly cancels asymmetric absorption. In the following we shall focus on the bulk interior of the star (below the layer where neutrinos decouple from the matter), where $U_{E}$ for $\nu_{e}$ has the Fermi-Dirac form with chemical potential $\mu_{\nu_{e}}$, while $\nu_{\mu}$ and $\nu_{\tau}$ have zero chemical potential. 
For a given neutrino species $i$, the total opacity can be written in the form

$$
\kappa_{E i}^{(t)}=\kappa_{E i}^{(\mathrm{abs})}+\kappa_{E i}^{(\mathrm{abs})}=\kappa_{i}\left(\frac{E}{E_{0}}\right)^{2}
$$

( $E_{0}$ is a fiducial energy). In general, because of nucleon degeneracy and/or neutrino degeneracy, there can be additional $E$-dependence in the prefactor $\kappa_{i}$ (Burrows \& Lattimer 1986). In these cases, we evaluate $\kappa_{i}$ at an appropriate mean neutrino energy. If we make a further assumption that $\nu_{e}$ and $\bar{\nu}_{e}$ have the same form of opacities (Burrows \& Lattimer 1986), then an analytical expression for the total energy flux (integrated over neutrino energy) associated with $\nu_{e}$ and $\bar{\nu}_{e}$ can be obtained:

$$
\mathbf{F}_{\nu_{e}}+\mathbf{F}_{\bar{\nu}_{e}}=-\frac{c E_{0}^{2}}{6 \pi^{2} \rho \kappa_{\nu_{e}}} \nabla\left[T^{2}\left(\frac{\eta_{\nu_{e}}^{2}}{2}+\frac{\pi^{2}}{6}\right)\right]+\frac{1}{2 \pi^{2}} \beta_{\nu_{e}} \epsilon c T^{4}\left(\frac{\eta_{\nu_{e}}^{3}}{3}+\frac{\pi^{2} \eta_{\nu_{e}}}{3}\right) \hat{\mathbf{B}}
$$

where we have defined

$$
\eta_{\nu_{e}} \equiv \frac{\mu_{\nu_{e}}}{T}, \quad \beta_{\nu_{e}} \equiv \frac{\kappa_{\nu_{e}}^{(\mathrm{sc})}}{\kappa_{\nu_{e}}^{(t)}}, \quad \epsilon \equiv \epsilon_{\nu_{e}} \equiv\left(\epsilon_{\mathrm{out}}-\epsilon_{\mathrm{in}}\right)_{\nu_{e}}
$$

In deriving Eq. (11) we have used the relation $\mu_{\bar{\nu}_{e}}=-\mu_{\nu_{e}}$ and $\epsilon_{\bar{\nu}_{e}}=-\epsilon_{\nu_{e}}$. Similarly, the net electron lepton number flux is given by

$$
\mathbf{S}_{\nu_{e}}-\mathbf{S}_{\bar{\nu}_{e}}=-\frac{c E_{0}^{2}}{6 \pi^{2} \rho \kappa_{\nu_{e}}} \nabla\left(T \eta_{\nu_{e}}\right)+\frac{1}{3 \pi^{2}} \beta_{\nu_{e}} \epsilon c T^{3}\left(\frac{\eta_{\nu_{e}}^{2}}{2}+\frac{\pi^{2}}{6}\right) \hat{\mathbf{B}}
$$

We shall group $\nu_{\mu}, \bar{\nu}_{\mu}, \nu_{\tau}$ and $\bar{\nu}_{\tau}$ together and denote them as $\nu_{\mu}$. With opacities in the form of Eq. (10), the total energy flux carried by these neutrinos is given by

$$
\mathbf{F}_{\nu_{\mu}}=-\frac{c E_{0}^{2}}{18 \rho \kappa_{\nu_{\mu}}} \nabla T^{2}
$$

As noted earlier, the asymmetry associated with $\mu$-type neutrinos completely cancels out.

With the relevant fluxes given above, the equations governing the thermal evolution of a proto-neutron star can be written as

$$
\begin{aligned}
\frac{\partial Y_{L}}{\partial t} & =-\frac{1}{n} \nabla \cdot\left(\mathbf{S}_{\nu_{e}}-\mathbf{S}_{\bar{\nu}_{e}}\right), \\
\frac{\partial U}{\partial t} & =-\nabla \cdot \mathbf{F}=-\nabla \cdot\left(\mathbf{F}_{\nu_{e}}+\mathbf{F}_{\bar{\nu}_{e}}+\mathbf{F}_{\nu_{\mu}}\right) .
\end{aligned}
$$

Here $Y_{L}=n_{L} / n=Y_{e}+Y_{\nu}=\left(Y_{e^{-}}-Y_{e^{+}}\right)+\left(Y_{\nu_{e}}-Y_{\bar{\nu}_{e}}\right)$ is the lepton number fraction $(n$ is the baryon number density), and $U$ is the internal energy density of the medium (a sum of contributions from neutrinos, electrons, positrons, photons, and nucleons). Note that inside the star, $\beta$-equilibrium holds to a good approximation, thus we have $\mu_{\nu_{e}}+\mu_{n}=\mu_{p}+\mu_{e}-1.293 \mathrm{MeV}$ (Note that electrons are extremely relativistic, while nucleons are non-relativistic; $\mu_{n}, \mu_{p}$ do not 
include rest mass). This expression is used to relate $\mu_{\nu_{e}}$ to the physical parameters of the medium ( $\rho, T, Y_{e}$, etc.). Also, for simplicity, we have neglected gravitational contraction and have adopted a Newtonian treatment. A general relativistic treatment including proto-neutron star contraction should not change our results by more than a factor of two.

We assume a uniform magnetic field in the $z$-direction throughout the neutron star. When the asymmetry is small, we can expand $T(\mathbf{r}, t)$ and $Y_{L}(\mathbf{r}, t)$ as

$$
\begin{aligned}
T(\mathbf{r}, t) & =T_{0}(r, t)+\Delta T(r, t) \cos \theta, \\
Y_{L}(\mathbf{r}, t) & =Y_{L 0}(r, t)+\Delta Y_{L}(r, t) \cos \theta,
\end{aligned}
$$

and similarly for other relevant quantities. The angular dependence can then be factored out, and the evolutionary equations reduce to two (coupled) sets of equations involving the symmetric and asymmetric terms. We solve these equations numerically using an explicit finite difference code.

Our model neutron star has a mass $M=1.38 M_{\odot}$ and a radius $R=11 \mathrm{~km}$, with a density profile resembling a $\Gamma=3$ polytrope (the central density is $\rho_{c}=8 \times 10^{14} \mathrm{~g} \mathrm{~cm}^{-3}$ ). The initial temperature and $Y_{L}$ profiles are shown in Fig. 1. In the stellar interior, we have $Y_{e}=0.28$, $Y_{L}=Y_{e}+Y_{\nu}=0.366$. The temperature is $8 \mathrm{MeV}$ in the inner core $\left(M \lesssim 0.7 M_{\odot}\right)$ and reaches 22 $\mathrm{MeV}$ in the outer core due to shock heating. These initial conditions are typical of those found in core collapse calculations (Burrows \& Lattimer 1986). The chemical potentials of various particles at the center are approximately given by $\mu_{n, p}=30.4\left(Y_{n, p} \rho_{14}\right)^{2 / 3} \mathrm{MeV}, \mu_{e}=239\left(Y_{e} \rho_{14}\right)^{1 / 3} \mathrm{MeV}$, and $\mu_{\nu}=301\left(Y_{\nu} \rho_{14}\right)^{1 / 3} \mathrm{MeV}$, with initial values $\mu_{n}=97.7 \mathrm{MeV}, \mu_{p}=52 \mathrm{MeV}, \mu_{e}=313 \mathrm{MeV}$, and $\mu_{\nu}=266 \mathrm{MeV}$. We assume that there is no asymmetry at $t=0$. We adopt the zero boundary condition $T=0$ at the stellar surface. Since the neutrino flux asymmetry mainly accumulates in the interior of the star, our crude treatment of the surface boundary condition is adequate.

Figure 1 depicts the $T_{0}, Y_{L 0}$ profiles and the asymmetric perturbations $\left(\Delta T\right.$ and $\left.\Delta Y_{L}\right)$ as functions of time for a magnetic field of strength $B_{14}=5$. The evolution of the $T_{0}$ and $Y_{L 0}$ profiles is similar to that found by Burrow \& Lattimer (1986): The deleptonization phase $(t \lesssim 10 \mathrm{~s})$ is dominated by the lepton number diffusion. In this early phase a heat wave moves inward, raising the core temperature - an effect analogous to the resistive heating of an electric conductor. This is followed by a thermal cooling phase in which the core temperature decreases gradually due to energy diffusion. The asymmetries in the temperature and lepton number profiles increase during the deleptonization phase, reaching a maximum at $t \sim 8 \mathrm{~s}$ (This behavior is analogous to that found in the toy problem discussed in $\S 3$ ). This growth of asymmetry is also reflected by the increase in the fractional asymmetry in luminosity $\Delta L_{z} / L$ (where $L$ is the luminosity, and $L_{z} / c$ is the net momentum along the magnetic axis carried away by the neutrinos per unit time) as shown in Fig. 2(b). As the neutron star becomes more and more lepton-depleted, the difference (in number and energy) between $\nu_{e}$ and $\bar{\nu}_{e}$ diminishes, and the asymmetry gradually declines.

In Fig. 2(c) we plot the asymmetry in the integrated neutrino luminosity $\left[\mathcal{E}(t)=\int_{0}^{t} d t L(t)\right]$ as a function of time. The fractional asymmetry in the total radiated neutrino energy is 
$\alpha=\Delta \mathcal{E}_{z} / \mathcal{E} \simeq 0.006 B_{14}$. This result is smaller than suggested by the toy problem (§3) mainly because the energy carried away by electron neutrinos during the deleptonization phase is only about $10 \%$ of the total neutrino energy released. In addition, geometric factors and cancellation of asymmetries associated with neutrons and protons also tend to reduce the total asymmetry in neutrino emission. Note that our result is much smaller than the crude estimate given by Horowitz \& Li (1997).

\section{Conclusion}

In this paper, we have identified the most efficient way of generating natal pulsar kicks based on asymmetric neutrino emission induced by magnetic fields. The key point of this mechanism is parity violation in weak interactions. The resulting pulsar kick velocity is

$$
V_{\text {kick }} \simeq 1000\left(\frac{\alpha}{0.084}\right)\left(\frac{E_{\text {tot }}}{10^{53} \mathrm{erg}}\right)\left(\frac{1.4 M_{\odot}}{M}\right) \simeq 214\left(\frac{\left\langle B_{z}\right\rangle}{10^{14} \mathrm{G}}\right)\left(\frac{E_{\mathrm{tot}}}{3 \times 10^{53} \mathrm{erg}}\right)\left(\frac{1.4 M_{\odot}}{M}\right) \mathrm{km} \mathrm{s}^{-1}
$$

where $E_{\text {tot }}$ is the total neutrino energy released by the proto-neutron star, and $\left\langle B_{z}\right\rangle$ is the (averaged) ordered component of the magnetic field in the star. We also note that the asymmetric $\nu_{e}$ and $\bar{\nu}_{e}$ emission from the proto-neutron star would give rise to asymmetric neutrino heating behind the stalled shock in the delayed supernova mechanism (LQ). Conceivably, this asymmetric heating could have some connections with the observed asymmetric supernova explosions.

Another related (but distinct) kick mechanism relies on the fact that $\nu_{e}$ and $\bar{\nu}_{e}$ absorption opacities near the neutrinosphere and the neutrino-matter decoupling region depends on the local magnetic field strength (due to quantization of $e^{-}$and $e^{+}$energy levels). If the magnitude of

magnetic field in the north pole is different from that in the south pole, then asymmetric neutrino flux can be generated. We have shown in a separate paper (Lai \& Qian 1997b) that this kick mechanism requires a much larger field strength than the mechanism considered in this paper.

It is plausible that magnetic fields stronger than $10^{15} \mathrm{G}$ can be generated in proto-neutron stars (Thompson \& Duncan 1993), and several pieces of evidence (albeit tentative) have been suggested in the literature (e.g., Vasisht \& Gotthelf 1997). While hydrodynamically-driven kick mechanisms may still be viable (depending on the magnitude of asymmetry seeded in the presupernova core; Goldreich et al. 1996), the possibility that a significant number of pulsars have kick velocities greater than $1000 \mathrm{~km} \mathrm{~s}^{-1}$ (e.g., Cordes \& Chernoff 1997) may well require large magnetic fields $\left(10^{15} \mathrm{G}\right)$ to be present in proto-neutron stars.

We thank Sterl Phinney for useful discussions at the early stage of this work. D.L. also thanks Ira Wasserman for valuable discussions. This work was started while D.L. held the Richard C. Tolman postdoctoral Fellowship at Caltech. Additional support was provided by NASA grant NAG 5-2756 and NSF grant AST-9417371. Y.Q. was supported by the David W. Morrisroe 
postdoctoral Fellowship at Caltech.

\section{REFERENCES}

Bisnovatyi-Kogan, G. S. 1993, Astron. Astrophys. Trans., 3, 287.

Burrows, A., Hayes, J., \& Fryxell, B. A. 1995, ApJ, 450, 830.

Burrows, A., \& Hayes, J. 1996, Phys. Rev. Lett., 76, 352.

Burrows, A., \& Lattimer, J. M. 1986, ApJ, 307, 178.

Chugai, N. N. 1984, Sov. Astron. Lett., 10, 87.

Cordes, J. M. 1986, ApJ, 311, 183.

Cordes, J. M., \& Chernoff, D. F. 1997, ApJ, submitted (astro-ph/9707308).

Cordes, J. M., Wasserman, I., \& Blaskiewicz, M. 1990, ApJ, 349, 546.

Dorofeev, O. F., et al. 1985, Sov. Astron. Lett., 11, 123.

Frail, D. A., Goss, W. M., \& Whiteoak, J. B. Z. 1994, ApJ, 437, 781.

Fryer, C., \& Kalogera, V. 1997, ApJ, in press (astro-ph/9706031).

Goldreich, P., Lai, D., \& Sahrling, M. 1996, in "Unsolved Problems in Astrophysics", ed. J. N. Bahcall and J. P. Ostriker (Princeton University press).

Hansen, B. M. S., \& Phinney, E. S. 1997, MNRAS, 291, 569 (astro-ph/9708071).

Harrison, P. A., \& Lyne, A. G. 1993, MNRAS, 265, 778.

Horowitz, C. J., \& Li, G. 1997, astro-ph/9705126.

Horowitz, C. J., \& Piekarewicz, J. 1997, hep-ph/9701214.

Janka, H.-T., \& Müller, E. 1996, A\&A, 306, 167.

Kaspi, V. M., et al. 1996, Nature, 381, 584.

Lai, D., Bildsten, L., \& Kaspi, V. M. 1995, ApJ, 452, 819.

Lai, D., \& Qian, Y.-Z. 1997a, to be submitted (LQ).

Lai, D., \& Qian, Y.-Z. 1997b, to be submitted.

Lorimer, D. R., Bailes, M., \& Harrison, P. A. 1997, MNRAS, 289, 592. 
Lyne, A. G., \& Lorimer, D. R. 1994, Nature, 369, 127.

McCray, R. 1993, ARA\&A, 31, 175.

Panagia, N. et al. 1996, ApJ, 459, L17.

Raffelt, G., \& Seckel, D. 1995, Phys. Rev. D, 52, 1780.

Thompson, C., \& Duncan, R. C. 1993, ApJ, 408, 194.

Vasisht, G., \& Gotthelf, E. V. 1997, ApJ, 486, L129.

Vilenkin, A. 1995, ApJ, 451, 700. 

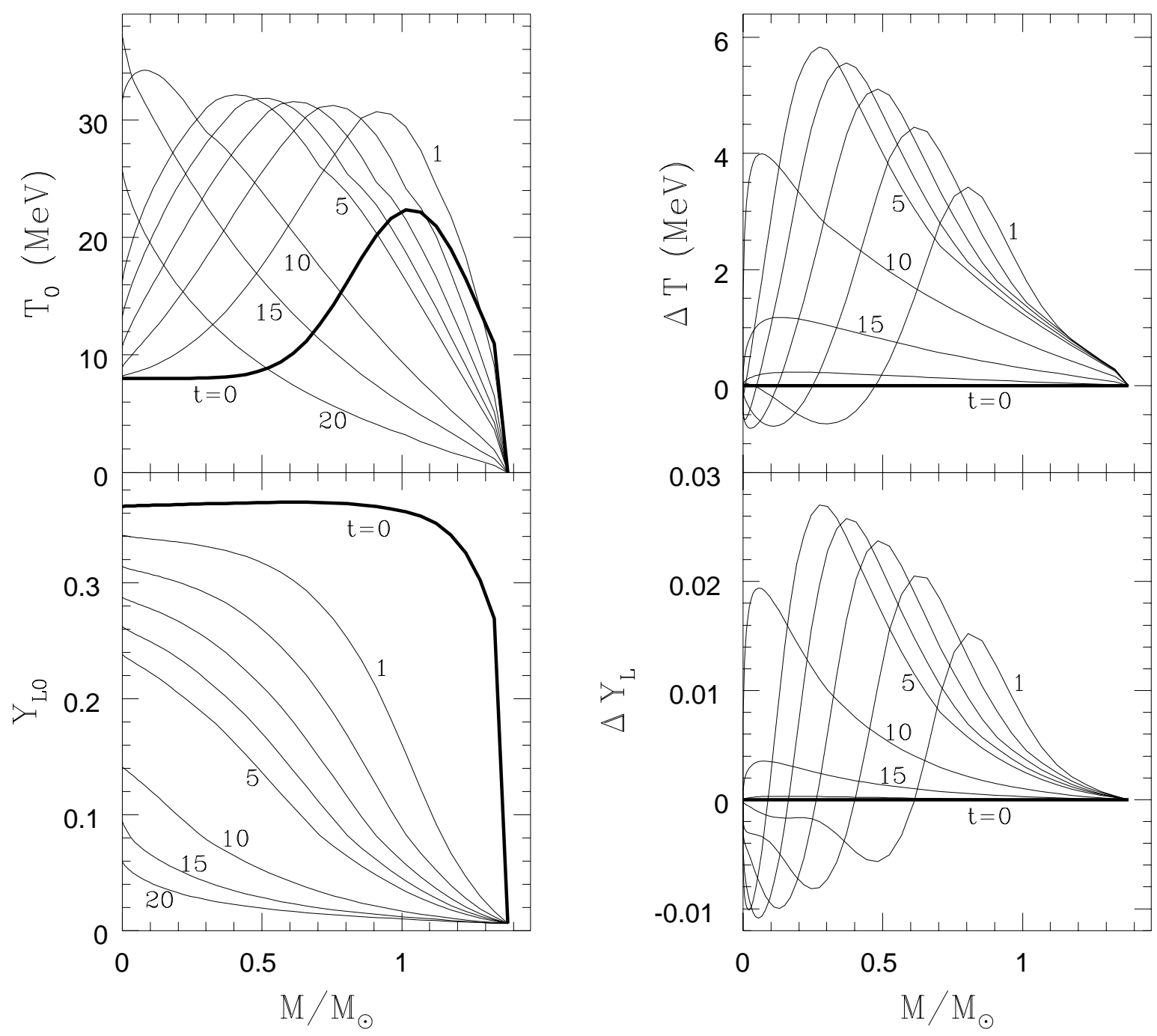

Fig. 1.- Thermal evolution of a proto-neutron star. The distributions of temperature and lepton number fraction (the left panels) and the asymmetric perturbations (the right panels) are shown as functions of time for a uniform magnetic field of strength $B_{14}=5$. 


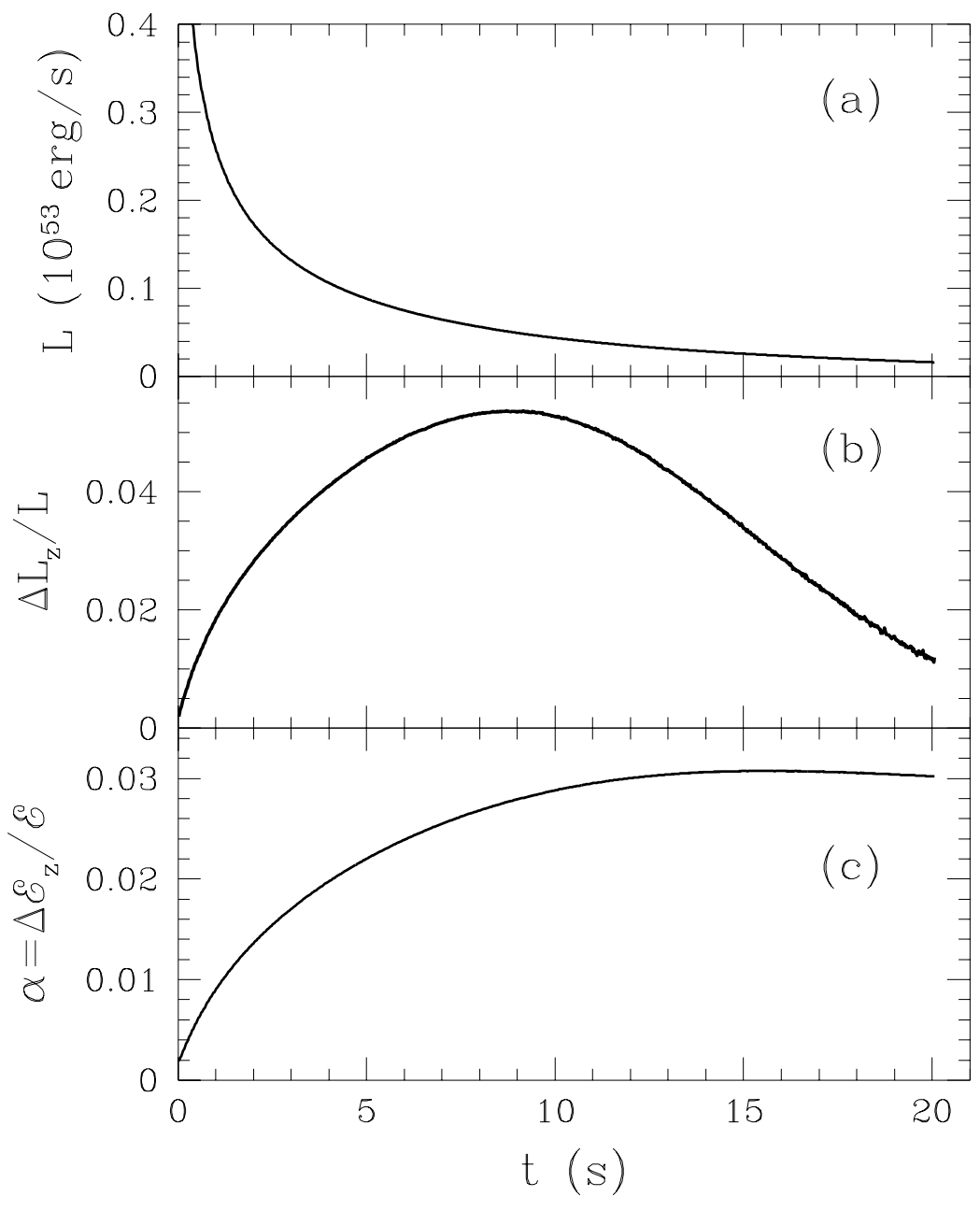

Fig. 2.- The time evolution of (a) total neutrino luminosity $L$, (b) fractional asymmetry in luminosity $\Delta L_{z} / L$, and (c) fractional asymmetry in the integrated luminosity $\alpha$, corresponding to the evolution depicted in Fig. 1. 